\title{
Logistic Formula in Biology and Its Application to COVID-19 in Japan
}

\author{
Akira Kokado* \\ Kobe Internationa Univerlsity, Kobe 658-0032, Japan \\ and \\ Takesi Saito $^{\dagger}$ \\ Department of Physics, Kwansei Gakuin University, Sanda 669-1337, Japan
}

\begin{abstract}
A logistic formula in biology is applied, as the first principle, to analyze the second and third waves of COVID-19 in Japan.
\end{abstract}

\section{Introduction}

The logistic formula is useful in the population problem in biology. It is closely related to the SIR model [1] in the theory of infection [2-9]. In previous papers [10-13] we have shown that the logistic formula can be approximately driven from the SIR model. In the present paper, however, we regard the logistic formula as the first principle, which is independent of the SIR model. We follow the observation that the removed number $R(t)$ in the SIR model behaves like the population in biology, that is, $R(t)$ is a sum of accumulated numbers of deaths and discharged.

In Sec. 2 we give the logistic formula for $R(t)$. Our policy is to use only data of removed by COVID-19 in Japan [14]. This formula will be applied to analyze the second and third waves in Secs. 3 and 4, respectively. These analyses revise results obtained in previous works $[12,13]$. The final section is devoted to concluding remarks. In Appendix we prepare error estimation formulas.

\section{The logistic formula}

In biology the logistic equation for a population $N(t)$ is given by

$$
\frac{d N(t)}{d t}=c I(t), \quad I(t)=A N(t)[B-N(t)]
$$

where $A, B$ and $c$ are some parameters. The solution is easily obtained as

$$
N(t)=\frac{B}{1+\exp (-z)}, \quad z \equiv A B c(t-T)
$$

*kokado@kobe-kiu.ac.jp

†tsaito@k7.dion.ne.jp

NOTE: This preprint reports new research that has not been certified by peer review and should not be used to guide clinical practice. 
Here $T$ gives a peak of $I(t)$, which is given by

$$
I(t)=\frac{A B^{2}}{2(1+\cosh z)},
$$

that is, $I(t)=A B^{2} / 4$.

The equation (2.1) can be regarded as the third equation of the SIR model ,

$$
\frac{d R(t)}{d t}=c I(t)
$$

if we identify $N(t)$ with $R(t)$, where $R(t)$ and $I(t)$ are the removed number and the infectious number, respectively, and $c$ the removed ratio. In previous works [10-13], our logistic formulas (2.2) and (2.3) have been driven approximately from the SIR model. In the present paper, however, we regard our logistic formulation as more fundamental rather than the SIR theory.

Let us rewrite Eq. (2.2) in notations $A=a / d, B=d$ and $N(t)=R(t)$ as follows:

$$
R(t)=\frac{d}{1+\exp (-z)}, \quad z \equiv a c(t-T),
$$

where $d$ is the final total removed number, e. g., $d=R(\infty)$. Eq. (2.5) can be expressed as

$$
-z=a c(T-t)=\ln F(t), \quad F=\frac{d}{R(t)}-1=\exp (-z) .
$$

Accordingly, for different times $t_{n}, t_{n+1}$ and $t_{n+2},(n=1,2, \ldots)$, we have

$$
\begin{aligned}
a c\left(t_{n+1}-t_{n}\right) & =\ln \frac{F\left(t_{n}\right)}{F\left(t_{n+1}\right)}, \\
a c\left(t_{n+2}-t_{n+1}\right) & =\ln \frac{F\left(t_{n+1}\right)}{F\left(t_{n+2}\right)} .
\end{aligned}
$$

When time differences in Eqs. (2.7) and (2.8) are equal, we have a useful formula for $d$

$$
\frac{F\left(t_{n}\right)}{F\left(t_{n+1}\right)}=\frac{F\left(t_{n+1}\right)}{F\left(t_{n+2}\right)}
$$

explicitly,

$$
\left(\frac{d}{R\left(t_{n}\right)}-1\right)\left(\frac{d}{R\left(t_{n+2}\right)}-1\right)=\left(\frac{d}{R\left(t_{n+1}\right)}-1\right)^{2}
$$

\section{Application to the second wave of COVID-19 in Japan}

Our logistic formula is applied to the second wave of COVID-19 in Japan. This provides a revise of previous work [12]

The $R(t)$ is the accumulated number of removed in the second wave in Japan, which is an average for 7 days in a middle at each $t$ with standard deviations, where $t$ is the date starting from June 20, 2020. The virus is now called the Tokyo type. We have subtracted the accumulated number 20507 on July 19 of removed in the first wave from that in the first and second waves. 
Table 1: Date t and the removed number $R(t)$ in the second wave in Japan [14]

\begin{tabular}{cc}
$\mathrm{t}$ & $\mathrm{D}(\mathrm{t})$ \\
\hline$t_{1}=$ Aug. 7 & $n_{1}=11377 \pm 2205$ \\
$t_{2}=$ Aug. 14 & $n_{2}=18949 \pm 2652$ \\
$t_{3}=$ Aug. 21 & $n_{3}=27455 \pm 2281$
\end{tabular}

Substituting data in the Table 1 into Eq. (2.10) with $n=1$, we have the equation for $d$

$$
\left(\frac{d}{11377}-1\right)\left(\frac{d}{27455}-1\right)=\left(\frac{d}{18949}-1\right)^{2} .
$$

to yield a solution

$$
d=45071
$$

From Eq. (2.7) with $n=1$ we get

$$
7 a c=\ln \frac{F_{1}}{F_{2}}=\ln 2.1482=0.7644, \quad a c=0.1092,
$$

with $F_{1} \equiv F\left(t_{1}\right)$ and $F_{2} \equiv F\left(t_{2}\right)$.

Substituting the result $a c=0.1092$ into Eq. (2.6), we have

$$
a c\left(T-t_{3}\right)=\ln F_{3}=\ln 0.6417=-0.4436 .
$$

with $F_{3} \equiv F\left(t_{3}\right)$, to yield to yield $T-t_{3}=-4.062$, so that

$$
T=t_{3}-4.062=\text { Aug. } 21-4.062=\text { Aug. } 17 \text {. }
$$

Error estimations for $d$ and $T$ can be seen from Appendix. By using relative errors.,

$$
\frac{\delta n_{1}}{n_{1}}=0.1938, \quad \frac{\delta n_{2}}{n_{2}}=0.1400, \quad \frac{\delta n_{3}}{n_{3}}=0.0831
$$

we have

$$
\frac{\delta d}{d}=0.0252, \quad \delta d=-1136, \quad \delta T=-3.87
$$

so that

$$
d=45071 \pm 1136, \quad \frac{d}{2}=22536 \pm 568, \quad T=\text { Aug. } 17 \pm 4 .
$$

The value of $d=45071$ on Aug.17 is plotted in Fig. 1. Values of $d$ for the other $n$ are also plotted in Fig. 1.

In Fig. 2 we draw a red curve of Eq. (2.5) for $R(t)$ calculated with the average value $d=44465$ and $a c=0.1106$ from Aug.13 to Aug.20, while the blue curve shows data for $R(t)$. Both lines coincide well in a region before Aug.27. The value of $\mathrm{d}$ should be constant as seen between Aug. 13 and Aug.20 within errors 1200 5000, but not before Aug.12. The deviation comes from the deviation between the red line and blue line in Fig. 2, where the red line is the calculated one of $R(t)$ and the blue line is its data. From this reason we abandon data outside of Aug.13 20.

To sum up, the second wave started from July.20, 2020, and peaked at Aug.17 with its total removed number $22536 \pm 568$. These calculated values should be compared with actual data that the peak date is around Aug.17 with its total removed number 22460. 


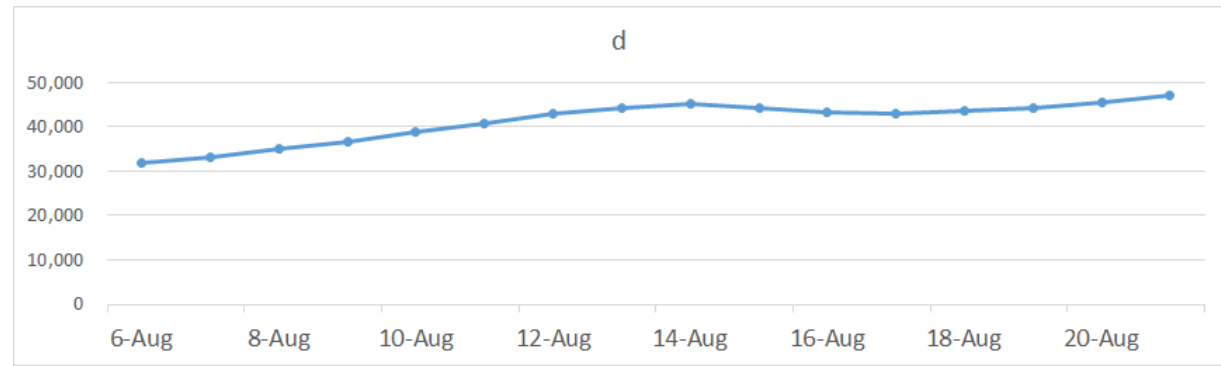

Figure 1: Blue points show values of $d$, which is defined by $d=R(\infty)$, e. g., the final total removed number, and is a solution of Eq. (2.10) for each $n$.

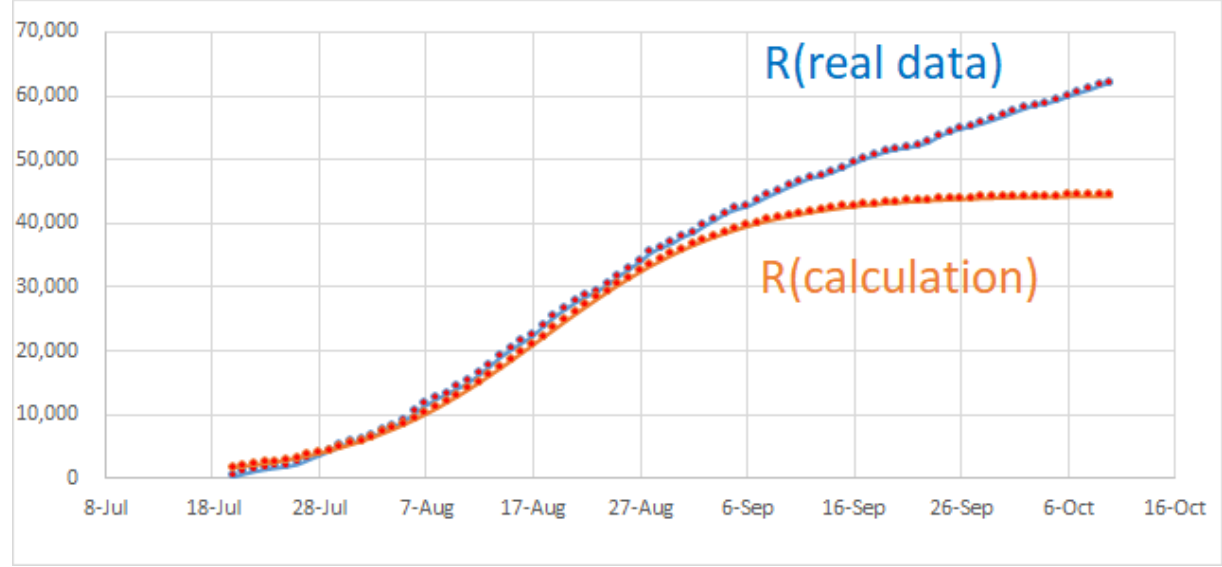

Figure 2: The red line is a calculated curve of $R(t)$, while the blue line shows data for $R(t)$. Both lines coincide well in a region before Aug. 27.

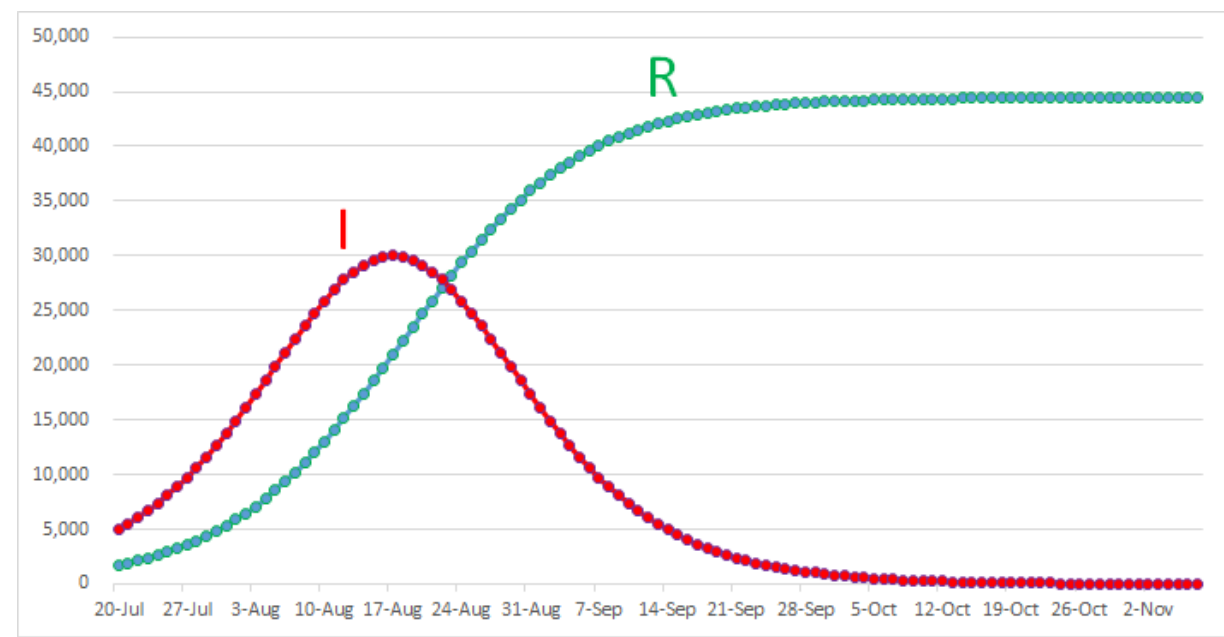

Figure 3: The blue line shows the calculated line of $R(t)$. The red line is the infectious number $I(t)$ with $d=4465$ and $a=2.698$.

\section{Application to the third wave of COVID-19 in Japan}

Our logistic formula is applied to the third wave of COVID-19 in Japan. This provides a revise of previous work [13] 
Table 2: Date and the removed number in the third wave in Japan [14]

\begin{tabular}{cc}
$\mathrm{t}$ & $\mathrm{D}(\mathrm{t})$ \\
\hline$t_{1}=$ Jan. 29 & $n_{1}=245292 \pm 11265$ \\
$t_{2}=$ Feb. 12 & $n_{2}=302185 \pm 5537$ \\
$t_{3}=$ Feb. 26 & $n_{3}=330669 \pm 3186$
\end{tabular}

The $R(t)$ is the accumulated number of removed in the third wave in Japan, which is an average for 7 days in a middle at each $t$ with standard deviations, where $t$ is the date starting from Oct. 11. We have subtracted the accumulated number 82810 of removed in the first and second waves from that in the first, second and third waves.

Substituting data in the Table 2 into Eq. (2.10) with $n=1$, we have the equation for $d$

$$
\left(\frac{d}{245292}-1\right)\left(\frac{d}{330669}-1\right)=\left(\frac{d}{302185}-1\right)^{2} .
$$

to yield a solution

$$
d=350329
$$

From Eq. (2.7) with $n=1$ we get

$$
14 a c=\ln \frac{F_{1}}{F_{2}}=\ln 2.6880=0.9888, \quad a c=0.0706 . .
$$

Substituting the result $a c=0.0701$ into Eq. (2.6), we have

$$
a c\left(T-t_{3}\right)=\ln F_{3}=\ln 0.0591=-2.8218 .
$$

to yield to yield $T-t_{3}=-39.97$, so that

$$
T=t_{3}-39.97=\text { Feb. } 26-39.97=\text { Jun. } 17 .
$$

Error estimations for $d$ and $T$ can be seen from Appendix. By using relative errors.,

$$
\frac{\delta n_{1}}{n_{1}}=0.046, \quad \frac{\delta n_{2}}{n_{2}}=0.018, \quad \frac{\delta n_{3}}{n_{3}}=0.0096
$$

we have

$$
\frac{\delta d}{d}=0.00946, \quad \delta d=3314.112, \quad \delta T=-0.035
$$

so that

$$
d=350329 \pm 3314, \frac{d}{2}=175164 \pm 1657, \quad T=\text { Jan. } 17 \pm 0 .
$$

The value of $d=350329$ on Feb. 12 is plotted in Fig. 4. Values of $d$ for the other $n$ are also plotted in Fig. 4.

In Fig. 5 we draw a red curve of Eq. (2.6) for $R(t)$ calculated with the average value $d=348008$ and $a c=0.0723$ from Jan. 28 to March 15, while the blue curve shows data for $R(t)$. Both lines coincide well in a region after Jan. 19. The value of $\mathrm{d}$ should be constant as seen after Jan. 24, but not before Jan. 24. The deviation comes from the deviation between the red line and blue line before Jan. 24 in Fig. 5, where the red line is the calculated one of $R(t)$ and the blue line is its data. From this reason we abandon data before Jan.24.

To sum up, the third wave started from Oct.11, 2020, and peaked at Jan. 17 with its total removed number $175165 \pm 1657$. These calculated values should be compared with actual data that the peak date is around Jan. 17 with its total removed number 177501. 


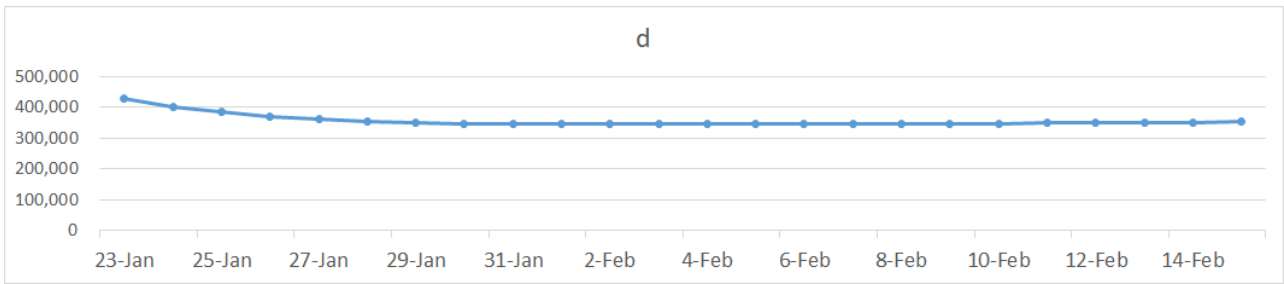

Figure 4: Blue points show values of $d$, which is defined by $d=R(\infty)$, e. g., the final total removed number, and is a solution of Eq. (2.10) for each $n$.

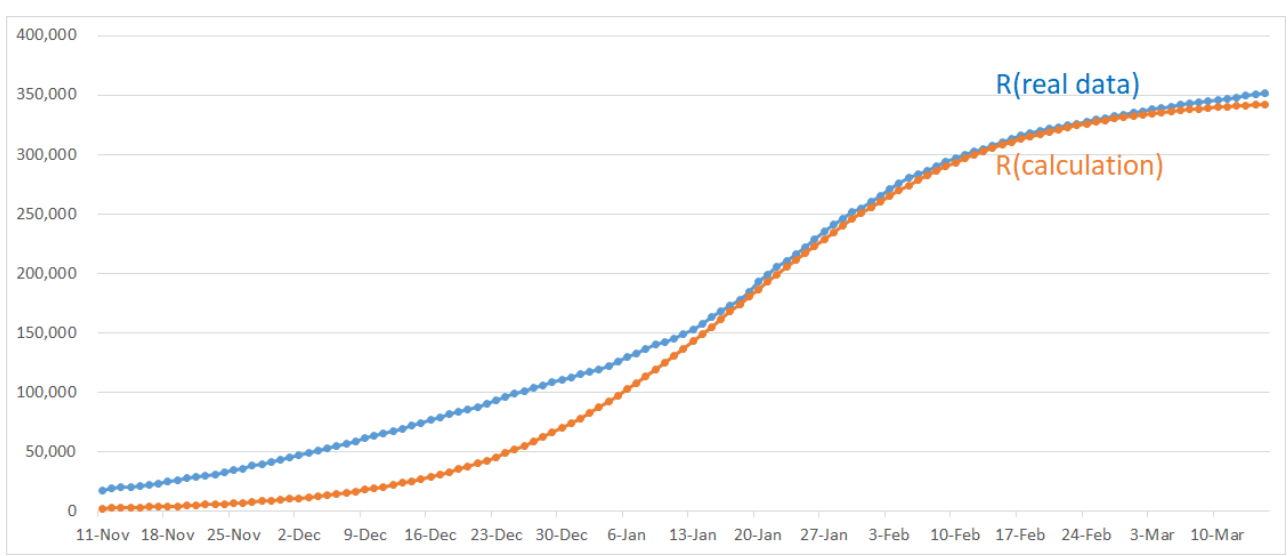

Figure 5: The red line is a calculated curve of $R(t)$, while the blue line shows data for $R(t)$. Both lines coincide well in a region after Jan. 24.

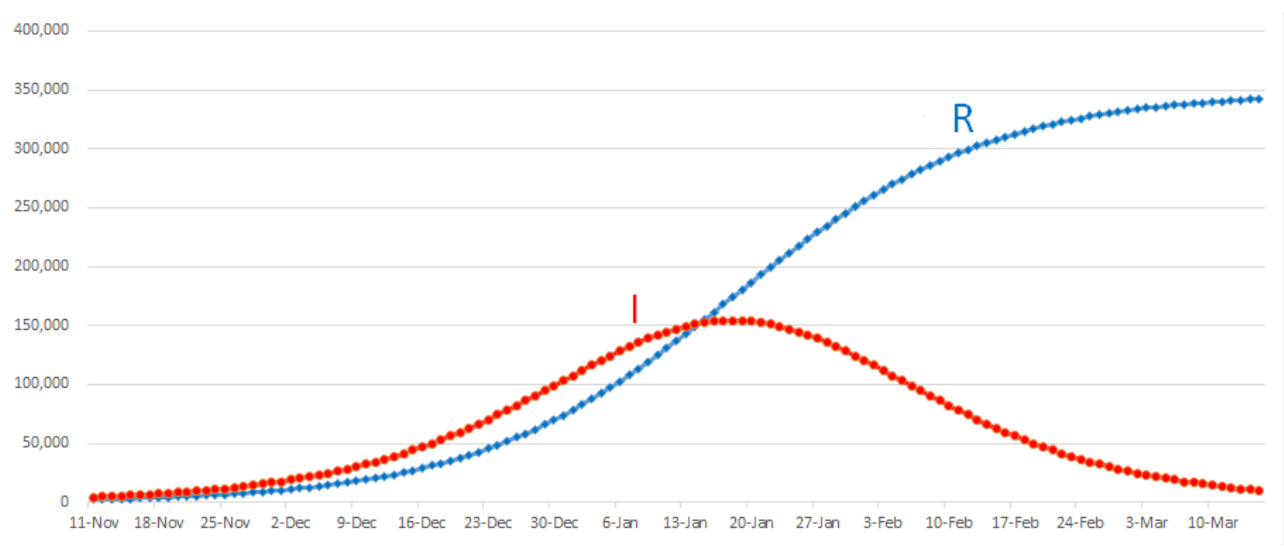

Figure 6: The blue line shows the calculated line of $R(t)$. The red line is the infectious number $I(t)$ with $d=348008$ and $a=1.763$.

\section{Concluding remarks}

The logistic formula in biology is applied, as the first principle, to analyze the removed number by the second and third waves of COVID-19 in Japan.

The second wave started from July.20, 2020, and peaked at Aug.17 with its total removed number $22536 \pm 568$. These calculated values should be compared with actual data that the peak date is around Aug.17 with its total removed number 22460.

The third wave started from Oct.11, 2020, and peaked at Jan. 17 with its total removed 
number $175165 \pm 1657$. These calculated values should be compared with actual data that the peak date is around Jan. 17 with its total removed number 177501. Results of the third wave have been obtained by using new data after the peak, Jan. 17. So, these are not a kind of prediction. However, we have succeeded to reproduce the peak data fairly well.

\section{Acknowledgement}

We would like to thank K. Shigemoto for many valuable discussions and big supports.

\section{Appendix Error estimation formulas}

From the formula $F_{1} F_{3}=F_{2}^{2}$ the relative error of removed number $d$ is driven as

$$
\frac{\delta d}{d}=\frac{F_{3}\left(1+F_{1}\right) \frac{\delta n_{1}}{n_{1}}+F_{1}\left(1+F_{3}\right) \frac{\delta n_{3}}{n_{3}}-2 F_{2}\left(1+F_{2}\right) \frac{\delta n_{2}}{n_{2}}}{F_{1}+F_{3}-2 F_{2}} .
$$

Secondly, the equation for the peak day $T, a c\left(T-t_{3}\right)=\ln F_{3}$, yields a formula

$$
a c \delta T=\frac{\delta F_{3}}{F_{3}}=\frac{1+F_{3}}{F_{3}}\left(\frac{\delta d}{d}-\frac{\delta n_{3}}{n_{3}}\right),
$$

from which one can estimate $\delta T$.

\section{References}

[1] W. O. Kermack and A.G. McKendrick, "A Contribution to the Mathematical Theory of Epidemics", Proceedings of the Royal Society A115 , 700-721 (1927)..

[2] W. D. Murray, "Epidemic models and the dynamics of infectious diseases", Mathematical Biology 42, 610-650 (1993).

[3] H. Hethcote, "The Mathematics of Infectious Diseases", SIAM Review 42, 599-653 (2000).

[4] M.J. Keeling and L. Danon, "Mathematical modelling of infectious diseases ", British Medical Bulletin 92, 33-42 (2009).

[5] S. Pathak, A. Maiti and G.P. Samanta, "Rich dynamics of an SIR epidemic model", Nonlinear Analysis: Modelling and Control 15, 71-81 (2010).

[6] T. Harko, F.S.N. Lobo, M.K. Mak, "Exact analytical solutions of the SusceptibleInfected-Recovered (SIR) epidemic model and of the SIR model with equal death and birth rates", Applied Mathematics and Computation 236, 184194 (2014).

[7] J.C. Miller, "Mathematical models of SIR disease spread with combined non-sexual and sexual transmission routes", Infectious Disease Modelling 2, 3555 (2017).

[8] R. Sameni, "Mathematical Modeling of Epidemic Diseases; A Case Study of the COVID19 Coronavirus", [arXiv:2003.11371 [q-bio.PE]](2017).

[9] P-F. Verhulst, "Notice sur la loi que la population poursuit dans son accroissement", Correspondance Mathmatique et Physique 10, 113-121 (1838).

[10] T. Saito and K. Shigemoto, "A Logistic Curve in the SIR Model and Its Application to Deaths by COVID-19 in Japan ", [medRxiv 10.1101/2020.06.25.20139865] (2020).

[11] Paul A. Reiser, “Modified SIR Model Yielding a Logistic Solution” , arXiv:2006.01550v1[q-bio.PE]. 
[12] T. Saito, "An Application of Logistic Formula to Deaths by COVID-19 in Japan", [medRxiv 10.1101/2020.09.15.20195081](2020).

[13] A. Kokado and T. Saito, "A Logistic Formula and Its Application to Deaths by the Third Wave of COVID-19 in Japan", [medRxiv 10.1101/2021.01.30.21250827](2021).

[14] Data published from the Ministry of Health, Labor and Welfare of Japan. See for example, "Summary of the New Coronavirus Infection "(in Japanese), https://hazard.yahoo.co.jp/article/20200207; "Status of the Domestic New Coronavirus Infection" (in Japanese), https://toyokeizai.net/sp/visual/tko/covid19/. 